\title{
HERRAMIENTAS E INDICADORES SEO: CARACTERÍSTICAS Y APLICACIÓN AL ANÁLISIS DE CIBERMEDIOS
}

\section{SEO tools and indicators: characteristics and application to online media analysis}

\author{
Lucía García-Carretero, Lluís Codina, Javier Díaz-Noci y Mar Iglesias-García
}

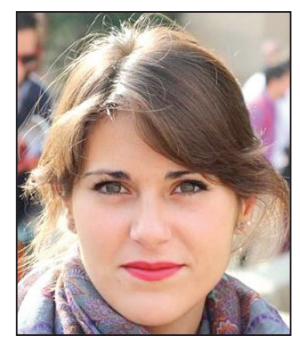

Lucía García-Carretero es graduada en periodismo por la Universidad de Valladolid y master en comunicación social por la Universitat Pompeu Fabra (UPF). Forma parte del personal docente investigador en la UPF con una beca de investigación. Es miembro del Grupo de Investigación en Periodismo (GRP) y del Grupo de Investigación en Documentación Digital y Comunicación Interactiva (DigiDoc). Sus líneas de investigación se centran en el estudio de la comunicación política en internet y en el análisis de estrategias comunicativas electorales en las redes digitales. http://orcid.org/0000-0002-1414-3921

Universidad Pompeu Fabra, Departamento de Comunicación Roc Boronat, 138. 08018 Barcelona, España lucia.garcia@upf.edu

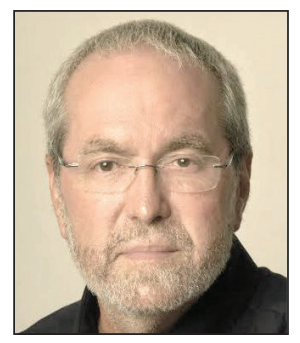

Lluís Codina es profesor de la Universitat Pompeu Fabra de Barcelona. Imparte docencia en la Facultad de Comunicación, en los grados de periodismo y de comunicación audiovisual. Es coordinador del Master universitario en comunicación social (MUCS) del Departamento de Comunicación. Forma parte del staff académico e imparte docencia también en los masters universitarios online en documentación digital y en buscadores del Instituto de Educación Continua. Es miembro promotor del Grupo de Investigación en Documentación Digital y Comunicación Interactiva (DigiDoc) y coordinador de su Seminario de Investigación.

http://orcid.org/0000-0001-7020-1631

Universidad Pompeu Fabra, Departamento de Comunicación Roc Boronat, 138. 08018 Barcelona, España Iluis.codina@upf.edu

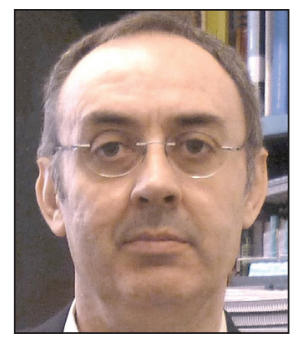

Javier Díaz-Noci es profesor en la Universitat Pompeu Fabra. Catedrático de universidad, fue profesor de la Universidad del País Vasco, ha sido profesor visitante de las universidades de Oxford (Reino Unido) y Federal de Bahía (Brasil). Licenciado en comunicación, diploma de estudios avanzados en derecho y doctor en historia, es autor de numerosos libros y artículos sobre periodismo en internet, historia del periodismo y derecho de autor de la obra periodística.

http://orcid.org/0000-0001-9559-4283

Universidad Pompeu Fabra, Departamento de Comunicación Roc Boronat, 138. 08018 Barcelona, España javier.diaz@upf.edu

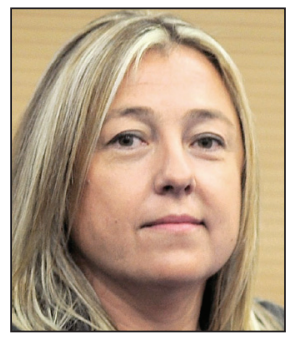

Mar Iglesias-García es periodista y profesora del Departamento de Comunicación y Psicología Social de la Universidad de Alicante. Imparte docencia en el grado de publicidad y RRPP y en el grado de turismo. Colabora en el proyecto de investigación Audiencias activas y periodismo. Interactividad, integración en la web y buscabilidad de la información, financiado por el Ministerio de Economía y Competitividad. Desde 2010 es directora del ciberperiódico Comunic@ndoUA. http://orcid.org/0000-0001-7926-5746

Universitat d'Alacant, Departamento de Comunicación y Psicología Social Carretera de San Vicente del Raspeig, s/n. 03690 San Vicente del Raspeig (Alicante), España mar.iglesias@gcloud.ua.es 


\section{Resumen}

La búsqueda es una de las actividades centrales en el mundo digital y, por tanto, uno de los elementos clave en el análisis de cibermedios, ya que una parte de sus audiencias y de sus ingresos procede de las páginas de resultados de los buscadores (SERP). En este trabajo, presentamos algunas de las herramientas de análisis de posicionamiento SEO más utilizadas con el fin de considerar su aplicación en estudios académicos sobre cibermedios. Aplicamos los nueve indicadores más importantes de estas herramientas a la página principal de cuatro cibermedios generalistas españoles con el fin de estimar su viabilidad como indicadores alternativos al PageRank y otros indicadores de Google.

\section{Palabras clave}

Medios; Cibermedios; Periódicos; SEO; Search engine optimization; SERP; Search engine results page; Búsquedas; PageRank; Posicionamiento web; Moz; Majestic; Ahref; Alexa.

\section{Abstract}

Searching is one of the core activities in the digital world and therefore, it is one of the key elements in the analysis of digital media since part of their audiences and revenues comes from the so called search engine result page (SERP). In this paper we present some of the most used analyzing tools in search engine optimization (SEO) in order to consider their application in online media research studies. We apply the nine most important indicators of these tools to the home page of four Spanish general online media in order to assess its viability as alternative indicators to PageRank and other Google indicators.

\section{Keywords}

Media; Digital Media; Newspapers; SEO; Search engine optimization; SERP; Search engine results page; Web search; PageRank; Moz; Majestic; Ahref; Alexa.

García-Carretero, Lucía; Codina, Lluís; Díaz-Noci, Javier; Iglesias-García, Mar (2016). “Herramientas e indicadores SEO: características y aplicación para análisis de cibermedios”. El profesional de la información, v. 25, n. 3, pp. $497-504$.

http://dx.doi.org/10.3145/epi.2016.may.19

\section{Introducción}

Hace tiempo que la búsqueda es una de las actividades centrales en el mundo digital, y esto afecta también a los cibermedios de manera específica. Una parte del tráfico, y por tanto una parte de sus audiencias, procede de las páginas de resultados de los buscadores o SERP (search engine result page) (Phillips; Yang; Djamasbi, 2013; Giomelakis; Veglis, 2015; Gonzalo; Codina; Rovira, 2015).

La omnipresencia de los buscadores entre las actividades de los internautas ha provocado a su vez el surgimiento de una industria centrada en el posicionamiento web. Se trata de un sector conocido como SEO (search engine optimization) (Vállez et al., 2010; Prat, 2012). Las empresas de este sector ofrecen herramientas de análisis, realizan auditorías y proponen recomendaciones para que las páginas de los sitios web queden bien posicionadas en las SERP (Büttcher; Clarke; Cormack, 2010; Codina, 2014).

Se trata de un sector que ya se puede considerar maduro y en el cual existe un buen número de empresas consolidadas por la capacidad de sus herramientas de análisis. Hay un amplio consenso entre los analistas en señalar como parte principal de este sector a empresas como Moz, Majestic, Alexa y Ahref (Virginia Tech University Libraries, s.d.). Tales empresas hace tiempo que proporcionan algunos de los softwares de análisis SEO más utilizados por los responsables de los sitios web que desean analizar no solamente su propia presencia en la Web, sino también la de sus competidores.

Sin embargo, pese a su amplia utilización en el mundo de las empresas, no existe apenas evidencia de su aplicación para estudios académicos sobre cibermedios (Palacios; DíazNoci, 2009; Codina et al., 2014; Masip, 2015; Freixa, 2015). En este trabajo nos hemos planteado por tal motivo considerar la validez y fiabilidad potencial de estas herramientas para investigaciones sobre cibermedios (Gulli; Signorini, 2005; Rodríguez-Martínez; Codina; Pedraza-Jiménez, 2012; Iglesias-García; Codina, 2016).

Para ello se propone la siguiente pregunta de investigación:

¿Las herramientas de análisis SEO utilizadas por diversos sectores de la Web podrían ser utilizadas con la misma eficacia también para el estudio académico de los cibermedios?

La anterior pregunta conduce a los siguientes objetivos, a los que se intenta dar respuesta en este trabajo:

- determinar con la mayor precisión posible qué indicadores proporcionan estas herramientas de análisis;

- realizar una serie de pruebas con diversos cibermedios utilizando los indicadores de las empresas citadas;

- llevar a cabo un análisis comparativo de resultados que eventualmente permita realizar estimaciones sobre la validez de las aplicaciones y poder así dar respuesta a la pregunta de investigación.

\section{Sistemas seleccionados}

Del análisis de webs relacionadas con la industria del SEO se obtienen los nombres de las empresas más destacadas del sector. Se han seleccionado aquellas que proporcionan herramientas online con al menos una parte de sus indicadores disponibles en abierto: 
- Moz

- Majestic

- Ahref

- Alexa

Para responder la pregunta de investigación, se presentan las herramientas correspondientes a estas empresas y sus principales indicadores. La razón de utilizar éstas responde a este doble cruce de características:

- forman parte del grupo de empresas de ámbito internacional más importantes, de acuerdo con la mayoría de los estudios del sector;

- estas empresas proporcionan versiones de acceso libre de sus programas.

A continuación se presentan los indicadores a partir de la información recogida en sus sitios web con el fin de conocer sus características y poder estimar su operatividad y fiabilidad. Finalmente se ha analizado cada cibermedio obteniendo información para cada uno de los indicadores definidos.

Podríamos preguntarnos por qué no utilizar también -o incluso, por qué no utilizar únicamente- los indicadores que proporciona Google. En el pasado era algo relativamente común y solía utilizarse una combinación de tres indicadores que resultó muy eficaz para caracterizar el perfil de un sitio: el PageRank (PR) y la aplicación de los comandos site y link a un dominio, como en site:www.elpais.com y link:www.elpais, por ejemplo.

La razón es que hace tres años que Google ha hecho inoperantes tales indicadores en la práctica debido a su falta de actualización. PageRank ha dejado de actualizarse hace más de un año y hace tiempo que se han documentado constantes inconsistencias en los resultados de los comandos indicados (Rovira, 2008). Aun así, nada impide a quien lo desee unir esta clase de indicadores a los que proporcionan webs como las que vamos a considerar a continuación.

Una página es tanto más importante cuantos más enlaces reciba de páginas que a su vez sean importantes

En todo caso, las empresas de análisis SEO optaron por elaborar sus propias variaciones del PageRank de Google. Originalmente suponemos que para poder ofrecer un producto diferenciador, pero desde hace algún tiempo como necesidad imperiosa dada la falta de actualización de PageRank (Page et al., 1999; Morato et al., 2013).

Tales variaciones toman como base la combinación de dos aspectos que también estaban presentes en el algoritmo del PageRank original:

- la suma de los enlaces de entrada (backlinks) de una página o dominio, según la cual una página es tanto más importante cuantos más enlaces recibe;

- la calidad de tales enlaces, que se calcula en función de la importancia de las páginas de las que proceden los enlaces.

Debido a esta doble combinación, una página es tanto más importante cuantos más enlaces reciba de páginas que a su vez sean importantes. Esto implica que cada enlace traslada una cantidad de "importancia" al que cabe asignarle un valor numérico para poder hacer los cálculos. En el PageRank original era un número de 0 a 10 (aunque este número era una simplificación, seguramente de base logarítmica, del número real que era conocido solamente por Google). Este número o valor de PR se trasladaba a las páginas que eran enlazadas, pero dividido por el número de enlaces de salida. Si una página con un PR de 4 por ejemplo tenía 4 enlaces de salida, el valor de PR que trasladaba a la página enlazada era de 1 exactamente.

Los analistas estiman que en los últimos años Google ha añadido un considerable número de factores de ponderación a este cálculo original. Por ejemplo, enlaces procedentes de foros de debate apenas trasladan PR o incluso nada; enlaces procedentes de sitios que no mantienen una relación temática, trasladan menos valor, etc.

Las empresas del sector se han visto obligadas a desarrollar sus propias métricas, pero como la base conceptual del PR es considerada valiosa en sí misma, estas métricas son variaciones de lo que hemos expuesto. Por ello se presentan las empresas objeto de esta investigación y, dentro de cada una de ellas, consideraremos los indicadores correspondientes. Después los aplicaremos a la página principal de un grupo de cibermedios para poder realizar una cata o estimación de su capacidad de correlación, y por último, discutiremos las posibles aportaciones de estos indicadores al estudio de los cibermedios.

\section{Moz \\ https://moz.com}

Según la información que el sitio web proporciona, la empresa productora de esta herramienta, SEOMoz -denominada únicamente Moz desde 2013- fue creada en 2004 como una empresa de consultoría, y a partir de 2007 se centra en el desarrollo de software propio para el análisis de indicadores SEO.

Sus indicadores se basan en una tecnología propia que denominan-desde un punto de vista corporativo y propio del marketing- Tagfee Code (Transparent \& authentic, generous, fun, emphathetic and exceptional). Su primera aplicación fue Moz Pro: un conjunto de herramientas independientes de SEO cuyo objetivo es la mejora del ranking y la visibilidad en los motores de búsqueda.

En 2008 lanzó Mozscape para el rastreo de la Web y en 2010 reunió todos sus programas en un único paquete. SEOMoz integró la primera versión del análisis social en el producto y en 2012 adquirió Getlisted destinado a empresas locales y creó Moz Local. En 2013 lanzó al público Moz Analytics.

A principios de 2015 contaba con más de 35.000 clientes. En su versión gratuita o semi gratuita encontramos:

- Open Site Explorer y Moz Bar: para el análisis de SEO;

- My Business Listing y Categories: análisis de marketing local;

- Content Marketing: marketing de contenido;

- Followerwonk: análisis de Twitter.

Para esta investigación nos centramos en Open Site Explo- 


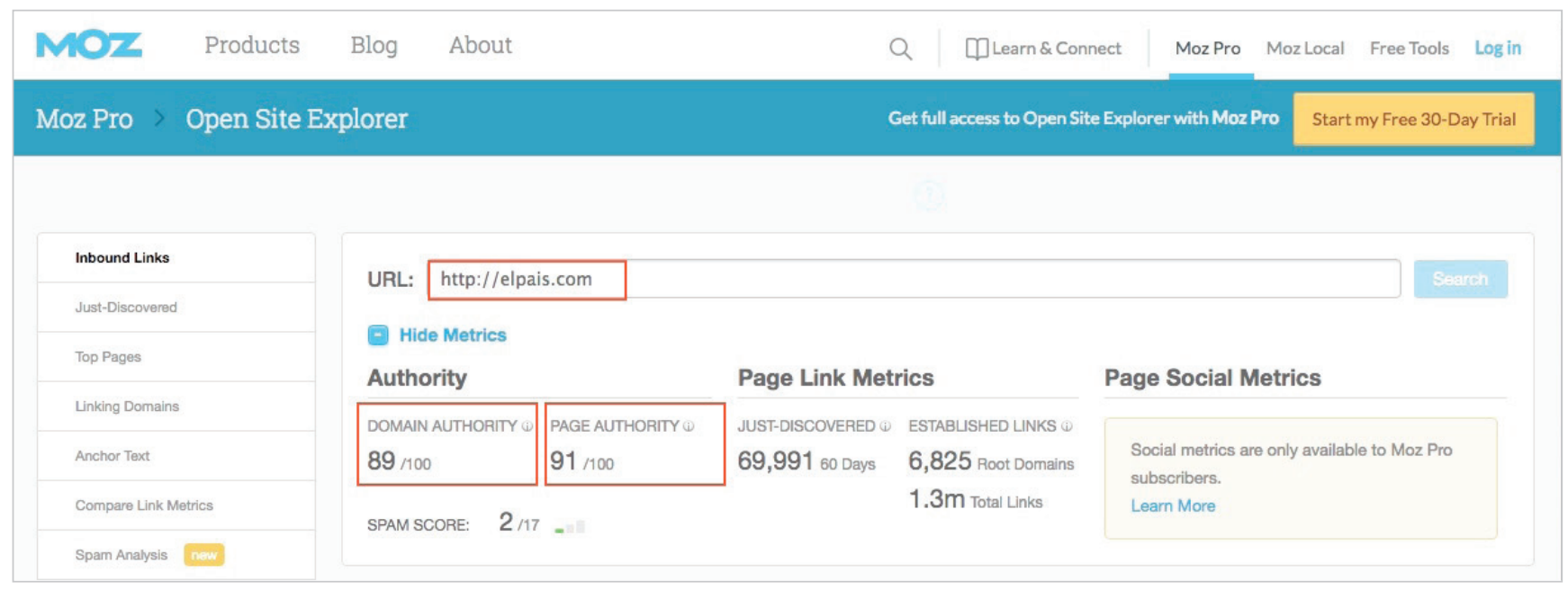

Imagen 1. Análisis de http://elpais.com en Moz

rer, la versión de uso abierto que proporciona información a través de dos indicadores principales: Domain authority y Page authority.

\section{Domain authority (DA)}

Autoridad del dominio indica de una forma sintética la importancia de un sitio. Según su propia definición "mide la fuerza de clasificación de dominios enteros o subdominios". Se refiere por tanto a la totalidad de un dominio, no a alguna de sus páginas individuales, ni siquiera a su página principal. El indicador consiste en una escala de 0 a 100. Dado que es una escala independiente de cualquier palabra clave, puede ayudar a predecir la situación de una web en los motores de búsqueda. Permite asimismo compararla con otras. Según señalan sus programadores, Moz calcula este indicador mediante una combinación de diversas métricas de enlaces en una única puntuación.

Domain authority mide la fuerza de clasificación de dominios enteros o subdominios, y Page authority mide la fuerza de clasificación de la página individual

Aunque conceptualmente se entiende que se refiere a una variación propia del PageRank, es decir, una métrica que agrega la cantidad y calidad de los enlaces de las páginas individuales, la empresa no facilita su forma de cálculo exacta. Esto puede parecer un inconveniente para su uso en estudios de tipo académico, pero lo cierto es que esta situación de relativa oscuridad sobre la forma exacta en la que se calcula una métrica es por desgracia habitual en el mundo del SEO.

\section{Page authority (PA)}

Autoridad de página, según Moz, "mide la fuerza de clasificación de la página individual". Se mide también mediante una puntuación en una escala de 0 a 100 que, como en el caso anterior, ayuda a predecir la situación de una página concreta en los motores de búsqueda basándose en los análisis de enlaces llevados a cabo por la citada empresa.

La pareja $D A$ y $P A$ es interesante porque, a diferencia de $P a$ geRank que únicamente se calcula a nivel de página -como en $P A-$, tenemos la posibilidad de conocer el potencial de visibilidad (o la autoridad, en términos Moz) de todo el dominio. Esto tiene sentido porque una noticia o una página concreta de un medio puede tener un PA muy bajo si no ha sido convenientemente optimizada, aunque el dominio del medio en cuestión tenga un DA muy alto.

\section{Majestic \\ https://es.majestic.com}

Surgió en 2004 para investigar y mapear internet y ha desarrollado su propia base de datos de análisis de enlaces. Según señala la propia empresa, Majestic revisa constantemente las páginas web y visita millones de urls al día. Cuenta con más de diez aplicaciones de análisis, como Search Explorer, Clique Hunter, o la utilizada en este trabajo, Site Explorer, que es su aplicación abierta. De Site Explorer se han utilizado los indicadores que presentamos a continuación:

\section{Trust flow estima la distancia de un domi- nio a una serie de dominios de referencia por su calidad y prestigio contrastado}

\section{Trust flow}

Flujo de confianza, uno de sus indicadores principales, indica la importancia o la calidad de un sitio y se mide en una escala de 0 a 100. Consiste en estimar la distancia de un dominio a una serie de dominios de referencia por su calidad y prestigio contrastado. Para ello recopila multitud de conjuntos de sitios que consideran de procedencia fiable, por ejemplo, medios de comunicación de prestigio, mediante una revisión manual de la Web global. Este proceso constituye la base del indicador Trust flow. 


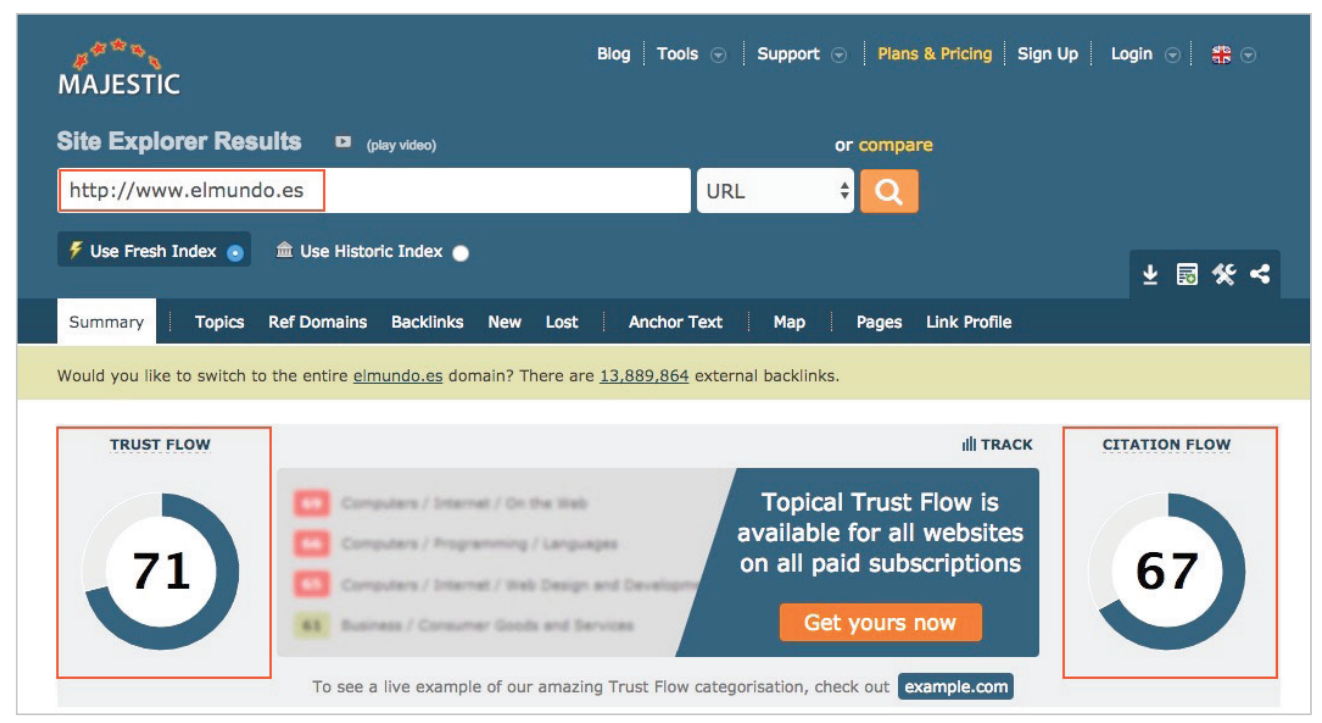

Imagen 2. Análisis de http://www.elmundo.es en Majestic centrarse en un sistema mucho más amplio que denominan 60TFlops System. Según indican en su web, su índice se actualiza cada 15 minutos con el análisis de los enlaces de entrada más recientes y mediante el proceso en cadena de más de 6 millones de webs cada día.

Entre los productos de esta empresa se encuentran Site Explorer y Backlink Checker, Content Explorer, Position Explorer, Keywords Explorer, Aherfs API, Domain Comparison, Batch Analysis y SEO Toolbar.
Los sitios estrechamente enlazados con un sitio de procedencia fiable obtendrán puntuaciones más altas. Por ejemplo, si deciden que los sitios .edu son de prestigio, un sitio que recibe un enlace directo de una universidad recibe una puntuación alta. Un sitio enlazado por este último, recibe algo menos de puntuación (porque está a dos pasos de distancia de .edu), y así progresivamente. En el extremo, sitios que están a mucha distancia de sitios fiables obtendrán puntuaciones mucho más bajas.

\section{Citation flow}

Flujo de menciones, como en los otros casos, es un indicador que recibe un valor entre 0 y 100 que sirve para medir el número de enlaces o, como señala la empresa, la "potencia" que posee el sitio web en cuestión. Sustituye al antiguo indicador ACRank (Rango de una mención). Puede servir para predecir el grado de influencia de un url en función del número de sitios que enlazan con él.

\section{Ahrefs https://ahrefs.com}

Es una empresa fundada en 2011, fecha en la que también presentaron al público su primera versión del programa Site Explorer, encargado del análisis de backlinks (enlaces de entrada) a partir de seis servidores propios iniciales, para más tarde
Como en los casos anteriores, nos centraremos en su aplicación abierta Site Explorer, a través de la cual se puede obtener un perfil detallado del backlink del sitio web o url. Así mismo ofrece información sobre qué sitios web enlazan con nuestro objeto de análisis y los términos utilizados en los anclajes o texto afectado por la etiqueta $<a>$ de los enlaces. De hecho esta aplicación permite llevar a cabo un seguimiento por horas del incremento del backlink del sitio web.

\section{Global rank}

Rango global indica la calificación o puntuación de un sitio web en función de un ranking mundial propio en base a parámetros de calidad. No facilita información detallada sobre su cálculo, por lo que es de suponer que lo componen a partir de una combinación de los dos indicadores que se definen a continuación y sobre los que tenemos algo más de información.

\section{Url rating}

Ratio de url mide la calidad y la cantidad de los backlinks, enlaces de entrada o retroenlaces del url concreto de una página web. En concreto, señala el impacto de todos los backlinks de una página en función del rango (calidad) y el número de enlaces que recibe.

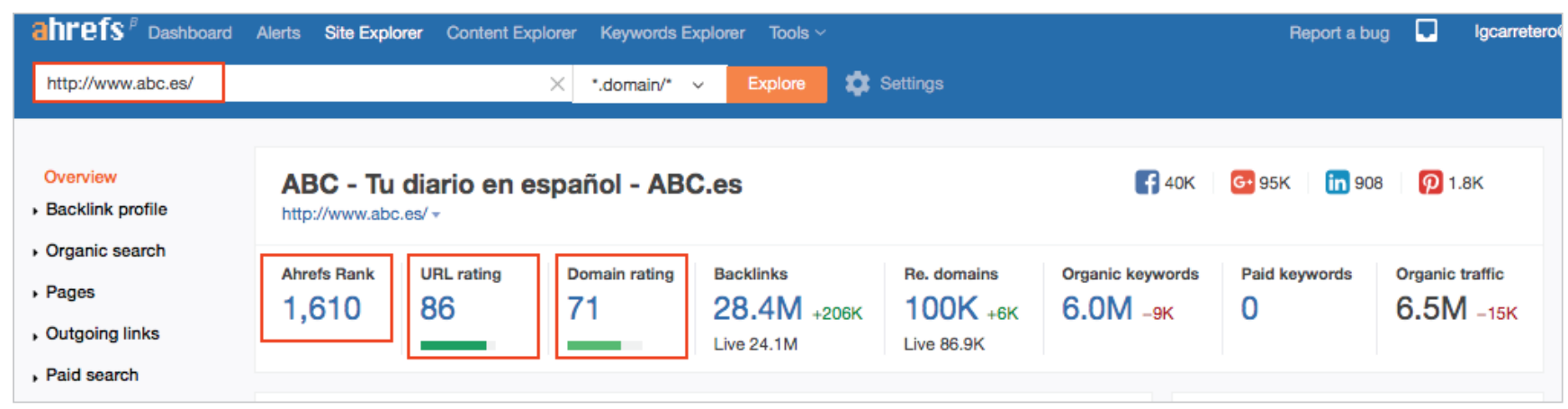

Imagen 3. Análisis de http://www.abc.es en Ahrefs 


\section{Domain rating}

El indicador Ratio de dominio mide la calidad y la cantidad de los backlinks (enlaces de entrada) del dominio de un sitio web. Se calcula a partir de los enlaces de las páginas individuales del conjunto del dominio a partir de una escala de ascendente 0 a 100 en función de la cantidad y calidad de los dominios vinculados. Url rating y Domain rating forman una pareja similar a $P A$ y $D A$ de $M o z$, a pesar de su diferente denominación.

\section{Alexa \\ http://www.alexa.com}

Surgió en 1996 y emplea datos procedentes de tres fuentes:

- dispone de acuerdos para analizar el tráfico de más de 2.500 servidores de control de tráfico de todo el mundo;

- reúne datos de sus fuentes directas en sitios web ya que ofrece la posibilidad a sus usuarios de instalar el script de Alexa y certificar sus métricas;

- obtiene datos de los varios millones de internautas que utilizan la toolbar de Alexa en sus navegadores y que puede descargarse de forma gratuita de su web.

Su rango de tráfico global mide cómo se relaciona una web con las demás. Ofrece una clasificación específica por países con el fin de conocer la posición de una web en relación con las de su territorio, actualizando esta información mensualmente. Las posibilidades de análisis que ofrece se organizan en productos como Site Overview -utilizado en esta investigación-Certified Site Metrics, Top Sites, Site Audits, Site Comparisons y Keyword Research.

\section{Global rank}

Rango global de Alexa se refiere al cálculo estimado de popularidad de un sitio web. Según la información que proporciona la empresa, se calcula mediante la combinación del promedio diario de visitantes que recibe una web y las páginas vistas de la misma durante los últimos tres meses. Su escala de medición se inicia en 1 para el sitio web con la mayor combinación de visitantes y páginas vistas de todo el mundo. Se actualiza diariamente.

\section{Local rank}

El indicador Rango local hace referencia al ranking de tráfico de cada país, es decir, se trata de una estimación de la popularidad de una web en un territorio nacional específico. Se calcula mediante la combinación del promedio de visitantes diarios en la web y las páginas vistas por los usuarios en el último mes. La escala de medición de este indicador se inicia en 1 siendo este el sitio web con una mayor combinación de visitantes y páginas vistas y, por tanto, mejor posición.

\section{Aplicación de indicadores a cibermedios}

Para tener una primera aproximación de la validez de los indicadores señalados, hemos optado por aplicar los 9 indicadores a 4 cibermedios españoles de referencia.

Se han analizado las páginas principales de 4 medios digitales con precedente en papel, de información general y de alcance nacional, que consideramos como más relevantes según datos de la OJD (2015) y de AIMC (2015), siendo conscientes de que no todos los medios se encuentran indexados:
- El país
- El mundo
- $A B C$
- La vanguardia

En la tabla 1 se presentan los datos obtenidos a partir de los 9 indicadores examinados anteriormente, organizados en función del medio objeto de estudio.

Se observa que existe cierta coherencia entre los datos proporcionados por los diferentes indicadores. Así según el Domain authority (ID1), es decir, según la relevancia de los dominios de cada cibermedio, observamos que tanto El país como El mundo son los más notables en relación con su dominio. Según este indicador, $A B C$ y La vanguardia ocuparían el tercer y cuarto puesto de la clasificación.

Por su parte, Page authority (ID2) -la relevancia o fuerza de la página individual del sitio web- muestra que las páginas de El país y la de El mundo son las mejor posicionadas, seguida por las de $A B C$ y La vanguardia. Como podemos comprobar a partir de la combinación de ambos indicadores del Site Explorer de Moz (ID1, ID2), existe cierta correspondencia entre la importancia tanto del dominio como de la página individual de los medios analizados.

En el grado de confianza o grado de calidad del sitio webTrust flow (ID3)-, El país es el medio de mayor calidad a partir de los estándares fijados por el indicador. A continuación $L a$ vanguardia, sigue $E I$ mundo y finalmente $A B C$.

Teniendo en cuenta el flujo de menciones (Citation flow, ID4) y, por tanto, el grado de influencia del url del ciberme-

Tabla 1. Aplicación de los indicadores a la página principal de los cibermedios analizados

\begin{tabular}{|c|c|c|c|c|c|c|c|c|c|}
\hline & $\begin{array}{c}\text { ID1 } \\
\text { Domain } \\
\text { Authority } \\
\text { (Moz) }\end{array}$ & $\begin{array}{c}\text { ID2 } \\
\text { Page } \\
\text { Authority } \\
\text { (Moz) }\end{array}$ & $\begin{array}{c}\text { ID3 } \\
\text { Trust Flow } \\
\text { (Majestic) }\end{array}$ & $\begin{array}{c}\text { ID4 } \\
\text { Citation } \\
\text { Flow } \\
\text { (Majestic) }\end{array}$ & $\begin{array}{c}\text { ID5 } \\
\text { Global } \\
\text { Rank } \\
\text { (Ahrefs) }\end{array}$ & $\begin{array}{c}\text { ID6 } \\
\text { URL Rating } \\
\text { (Ahrefs) }\end{array}$ & $\begin{array}{c}\text { ID7 } \\
\text { Domain } \\
\text { Rating } \\
\text { (Ahrefs) }\end{array}$ & $\begin{array}{c}\text { ID8 } \\
\text { Global } \\
\text { Rank } \\
\text { (Alexa) }\end{array}$ & $\begin{array}{c}\text { ID9 } \\
\text { Local Rank } \\
\text { (Alexa) }\end{array}$ \\
\hline elpais.com & 88 & 90 & 79 & 65 & 836 & 87 & 74 & 407 & 10 \\
\hline elmundo.es & 88 & 90 & 72 & 72 & 1.255 & 82 & 74 & 518 & 12 \\
\hline abc.es & 84 & 87 & 69 & 60 & 1.549 & 85 & 72 & 1.263 & 25 \\
\hline lavanguardia.com & 83 & 86 & 75 & 65 & 3.581 & 85 & 69 & 1.859 & 32 \\
\hline
\end{tabular}

(ID: identificación del indicador) 
dio en relación con el número de sitios web que enlazan con ella, notamos que el periódico que ocupa la primera posición es El mundo. Tanto El país como La vanguardia se encuentran igualados en menciones externas $y$, en último lugar, se sitúa $A B C$.

El Global rank (ID5) de Ahrefs lo interpretamos a partir de sus otros dos indicadores, el Url rating (ID6) y el Domain rating (ID7) ya que la empresa no facilita información suficiente sobre su cálculo. Así, observamos que según la calidad y la cantidad de los enlaces de entrada al url del medio (ID6), El país ocupa la primera posición, en segundo lugar encontramos empatados $A B C$ y $L a$ vanguardia y el cuarto lugar es para El mundo. Por su parte, analizando estos cibermedios a partir de los enlaces de entrada a sus dominios (ID7) el ranking varía ligeramente y El país ocupa la primera posición empatado con $E I$ mundo y les siguen $A B C$ y $L a$ vanguardia.

Los indicadores que Alexa ofrece, Global rank (ID8) y Local rank (ID9), es decir, popularidad de un sitio web a nivel global y local, permiten observar que la relevancia de los cuatro cibermedios sigue el mismo patrón, tanto fuera como dentro de nuestras fronteras. Entendemos, pues, que el cibermedio que mayor tráfico recibe, tanto en territorio español como fuera, es $E l$ país, seguido por $E I$ mundo, $A B C$ y, por último, La vanguardia.

\section{Discusión y conclusiones}

Dada la compleja naturaleza de los cibermedios en relación con otros sitios web, consideramos que uno de los aspectos más significativos a la hora de evaluar o medir el posicionamiento o visibilidad de un medio es el conjunto de factores OnPage. Se trata de un conjunto de factores bajo el control total del productor de la página dado que se centra en su contenido y su vinculación a enlaces y palabras clave (Codina et al., 2016).

Los indicadores contemplados en este artículo pueden sustituir la ausencia de indicadores actualizados y consistentes por parte de Google, como es sabido, el buscador más utilizado de nuestro país, y sin embargo el menos fiable a la hora de utilizar sus indicadores, como PageRank y los otros dos citados, por su obsolescencia y su falta de consistencia (Rovira, 2008).

Como se puede observar en la tabla 2, en el ranking general de cibermedios realizado a partir de los diferentes análisis existen alguna oscilación en las posiciones. No obstante, se puede ver que, en todas las herramientas de análisis, El país ocupa la primera posición -en el caso de Moz igualado con elmundo.es- y el resto de posiciones solamente varía para Majestic. Esto es así, no obstante, el hecho de que en el caso de Alexa los indicadores se basan en datos de tráfico y no en enlaces. En relación con estos datos de tráfico, algunos analistas señalan que puede existir cierto sesgo por parte de Alexa ya que su ranking cuenta con sitios asociados sobrerrepresentados y sitios insuficientemente representados (Norving, 2012), situación que en el caso de nuestras mediciones no ha podido constatarse.

La falta de transparencia en algunos indicadores debe entenderse como un problema relativo. Lo cierto es que no se conocen con detalle algunos de los cálculos, pero sí conocemos su fundamento conceptual, que se ha mostrado en los apartados precedentes y que, recordemos, se refieren al número y calidad de los enlaces que recibe una página o un sitio. Siendo a su vez el concepto de calidad referido o bien a sitios muy enlazados o a sitios próximos a otros de referencia (como sitios .edu). No existe en todo caso ninguna forma de medida mínimamente compleja que implique uso de estadísticas que no esté aquejada de problemas de definición, de problemas de toma de datos, o de ambos a la vez.

Por tanto, tampoco sería un escenario inédito entre los investigadores acostumbrados a tratar con resultados procedentes de algún tipo de minería de datos. En todo caso, la misma situación existía cuando PageRank era un instrumento vigente. Sabíamos el fundamento conceptual, pero ignorábamos los detalles de su cálculo. Y lo mismo sucede con algunos conocidos indicadores bibliométricos que utilizan bases de datos como Web of Science o Scopus: conocemos bien su fundamento conceptual, pero no sabemos los detalles procedimentales de su cálculo.

Lo que puede hacerse es, o bien tomar como indicadores para cada estudio los mismos de alguna de las herramientas (de esta manera, los posibles sesgos se aplican por igual), o combinar más de una y utilizar resultados ponderados.

Sí que destacan los indicadores que presenta Alexa, ya que son de total transparencia. Tanto su Global como su Local rank tienen bases estadísticas bien definidas en su sitio web que proceden de la combinación del número de visitas y del número de páginas vistas en promedio.

Además, cada una de las empresas consideradas aquí presenta otros indicadores que no hemos considerado pero que pueden ser sin duda de gran utilidad para estudios de cibermedios. Es también el caso de Alexa que, por ejemplo, proporciona indicadores como el Bounce rate (Tasa de rebote) que indica el porcentaje de usuarios que abandona un sitio sin hacer ningún clic.

En futuras investigaciones, estos indicadores pueden usarse para el análisis de hechos noticiables concretos, ya que pueden aplicarse a páginas concretas en lugar de a la página principal del medio y medir de esta forma la capacidad de

Tabla 2. Ranking de cibermedios según las herramientas seleccionadas

\begin{tabular}{|l|l|l|l|l|}
\cline { 2 - 5 } \multicolumn{1}{c|}{ Moz } & \multicolumn{1}{c|}{ Majestic } & \multicolumn{1}{c|}{ Ahrefs } \\
\hline $\mathbf{1}$ & elpais.com & elpais.com & elpais.com & elpais.com \\
\hline $\mathbf{2}$ & elmundo.es & lavanguardia.com & elmundo.es & elmundo.es \\
\hline $\mathbf{3}$ & abc.es & elmundo.es & abc.es & abc.es \\
\hline $\mathbf{4}$ & lavanguardia.com & abc.es & lavanguardia.com \\
\hline
\end{tabular}


difusión y de interacción de las audiencias con las noticias de cada cibermedio.

\section{Nota}

Este trabajo forma parte del proyecto Creación y contenido interactivo en la comunicación de información audiovisual: audiencias, diseño, sistemas y formatos. CSO2015-64955C4-2-R, del Programa Estatal de Investigación. Ministerio de Economía y Competitividad (España).

\section{Bibliografía}

Asociación para la Investigación en Medios de Comunicación (EGM). Audiencia de internet.

http://www.aimc.es/-Audiencia-de-Internet-en-el-EGM-.htm/

Büttcher, Stefan; Clarke, Charles L. A.; Cormack, Gordon V. (2010). "Web search". En: Büttcher, Stefan; Clarke, Charles L. A.; Cormack, Gordon V. Information retrieval: Implementing and evaluating search engines. Massachusetts: The MIT Press, pp. 507-563. ISBN: 9780262026512

Codina, Lluís (2014). "16 recursos de Google imprescindibles para el SEO de sitios periodísticos". Lluís Codina [UPF] docencia e investigación en comunicación y documentación digital, 27 septiembre.

http://www./luiscodina.com/seo-contenidos-actualidad

Codina, Lluís; Iglesias-García, Mar; Pedraza, Rafael; GarcíaCarretero, Lucía (2016). Visibilidad y posicionamiento web de informaciones periodísticas: el framework SEO-RCP. Barcelona: Serie editorial DigiDoc-UPF.

http://repositori.upf.edu/handle/10230/26040

Codina, Lluís; Pedraza-Jiménez, Rafael; Díaz-Noci, Javier; Rodríguez-Martínez, Ruth; Pérez-Montoro, Mario; Cavaller-Reyes, Víctor (2014). "Sistema articulado de análisis de cibermedios (SAAC): una propuesta sobre el qué y el cómo para estudiar los medios de comunicación digitales". Hipertext.net, n. 12

http://dx.doi.org/10.2436/20.8050.01.13

Freixa, Pere (2015). "Reportajes especiales en los cibermedios. Análisis de diez años de Premios Goya y Oscar Awards en Elpais.com (2005-2014)". El profesional de la información, v. 24, n. 3, pp. 291-300.

http://dx.doi.org/10.3145/epi.2015.may.09

Giomelakis, Dimitrios; Veglis, Andreas (2015). "Employing search engine optimization techniques in online news articles". Studies in media and communication, v. 3, n. 1, pp. 22-33.

http://dx.doi.org/10.11114/smc.v3i1.683

Gonzalo, Carlos; Codina, Lluís; Rovira, Cristòfol (2015). "Recuperación de información centrada en el usuario SEO: categorización y determinación de las intenciones de búsqueda en la Web". Index.comunicación, v. 5, n. 3, pp. 19-27.

http://journals.sfu.ca/indexcomunicacion/index.php/indexcomunicacion/article/view/197

Gulli, Antonio; Signorini, Alessio (2005). "The indexable web is more than 11.5 billion pages". En: The $14^{\text {th }}$ intl conf on World Wide Web (WWW'05), May 10-14, Chiba, Japan, pp. 902-903.

http://www.di.unipi.it/ qulli/papers/f692_gulli_signorini.pdf
Iglesias-García, Mar; Codina, Lluís (2016). “Los cibermedios y la importancia estratégica del posicionamiento en buscadores (SEO)". Opción. En prensa.

Masip, Pere (2015). “Medios de comunicación digitales en 2014: un balance alternativo". Informes ThinkEPI 2015 sobre documentación y comunicación, pp. 149-158. ISBN: 97884 60682097

Morato, Jorge; Sánchez-Cuadrado, Sonia; Moreno, Valentín; Moreiro-González, José-Antonio (2013). “Evolución de los factores de posicionamiento web y adaptación de las herramientas de optimización". Revista española de documentación científica, v. 36, n. 3, pp. 1-12.

http://dx.doi.org/10.3989/redc.2013.3.956

Norving, Peter (2012). "Alexa toolbar and the problem of experiment design".Peter@Norving.com.

http://norvig.com/logs-alexa.htm/

Oficina de Justificación de la Difusión (2015). Diciembre 2015. Tráfico nacional e internacional.

http://www.ojdinteractiva.es/medios-digitales

Page, Lawrence; Brin, Sergey; Motwani, Rajeev; Winograd, Terry (1999). The PageRank citation ranking: Bringing order to the Web (Techreport). Stanford: Stanford InfoLab. http://ilpubs.stanford.edu:8090/422

Palacios, Marcos; Díaz-Noci, Javier (eds.) (2009). Online journalism: Research methods: A multi-disciplinary approach in comparative perspective. Servicio Editorial de la Universidad del País Vasco: Bilbao. ISBN: 9788498601916 http://gjol.net/wp-content/uploads/2012/12/book-onlineresearch-eng.pdf

Phillips, Adrienne H.; Yang, Ruijiao; Djamasbi, Soussan (2013). "Do ads matter? An exploration of web search behavior, visual hierarchy, and search engine results pages". En: $46^{\text {th }}$ Hawaii intl conf on system sciences (Hicss), pp. 1563-1568. http://dx.doi.org/10.1109/HICSS.2013.181

Prat, Marie (2012). SEO-Posicionamiento de su sitio web en Google y otros buscadores. 2ㅇe ed. ENI: Barcelona. ISBN: 978 2746073227

Rodríguez-Martínez, Ruth; Codina, Lluís; Pedraza-Jiménez, Rafael (2012). "Indicadores para la evaluación de la calidad en cibermedios: análisis de la interacción y de la adopción de la web 2.0". Revista española de documentación científica, v. 35, n. 1, pp. 61-93.

http://dx.doi.org/10.3989/redc.2012.1.858

Rovira, Cristòfol (2008). "Datos numéricos en resultados de los buscadores". Anuario ThinkEPI, v. 2, pp. 76-78.

http://recyt.fecyt.es/index.php/ThinkEPI/article/view/32045

Vállez, Mari; Rovira, Cristòfol; Codina, Lluís; Pedraza-Jiménez, Rafael (2010). "Procedures for extracting keywords from web pages, based on search engine optimization". Hipertext.net, n. 8.

https://www.upf.edu/hipertextnet/en/numero-8/keywords extraction.html

Virginia Tech University Libraries (n.d.). Evaluating webpages for research. Evaluation internet information.

http://www.lib.vt.edu/instruct/evaluate 